Moroccan J. of Pure and Appl. Anal. (MJPAA)

Volume 7(3), 2021, Pages 375-384

ISSN: Online 2351-8227 - Print 2605-6364

DOI: $10.2478 / \mathrm{mjpaa}-2021-0025$

\title{
Some results about Lipschitz $p$-Nuclear Operators
}

\author{
Khedidja Bey ${ }^{1}$, Amar Belacel ${ }^{2}$
}

Aвstract. The aim of this paper is to study the onto isometries of the space of strongly Lipschitz $p$-nuclear operators, introduced by D. Chen and B. Zheng (Nonlinear Anal.,75, 2012). We give some new results about such isometrics and we focus, in particular, on the case $F=\ell_{p^{*}}$.

Mathematics Subject Classification (2010). Primary: 46A32, 46B04, 46B20; Secondary: 46B28, 47B10, 47L20. Key words and phrases. Isometries, Lipschitz operators, Lipschitz p-nuclear operators, Pointed metric space.

\section{Introduction}

In [11], Farmer and Johnson introduced a natural non linear version of $p$-summing operator, where the domain of such operators is a metric space that need not be a normed space. Since then, several authors have developed many works related to the classe of Lipschitz operator ideal [1], in the sense of creating a theory analogous to the classe of linear operator ideal [16]. For example see $[1,3,6,7,8,9,12]$ and the references therein, with a linear case that has already been introduced and studied by others.

In this paper, we contribute to the theory of non-linear of $p$-nuclear operators by extending the notion of the isometries of $p$-nuclear operators to Lipschitz case whose linear analogue has studied by Yousef and Khalil in [15]. This paper is organized as follows: In the second section, we gave some notations and Preliminaries interesting for our work. In the third one,

Received July 05, 2020 - Accepted: March 11, 2021.

(C) The Author(s) 2021. This article is published with open access by Sidi Mohamed Ben Abdallah University.

1,2 Laboratoire de Mathmatiques Pures et Appliques, University of Laghouat 03000, Laghouat, Algeria. e-mail ${ }^{1}$ : kh.bey@lagh-univ.dz (Corresponding Author),

e-mail²: a.belacel@lagh-univ.dz. 
we studied the Lipschitz version the isometric onto operators of $\mathcal{N}_{p}(E . F)$ [15]. In the last one, we continued in same direction with $F=\ell_{p^{*}}$, where we have given results, among others, concerning rank and atoms.

\section{Notation and Preliminaries}

For $1 \leq p \leq \infty, p^{*}$ denotes the exponent conjugate to $p$, that is $\frac{1}{p}+\frac{1}{p^{*}}=1$. $E, F$ are (real or complex) Banach spaces and $\mathcal{L}(E, F)$ denote the Banach space of all bounded linear operators from $E$ into $F$ with the operator norms. $T$ belong to $\mathcal{L}(E, F)$ is called an isometry if $\|T x\|=\|x\|$ for all $x \in E$.

The space $\operatorname{Lip}_{0}(X, F)$ is a Banach space of all Lipschitz operators $T$ from a pointed metric space $X$, metric space with a distinguished point denoted 0 , into $F$ such that $T(0)=0$, under the Lipschitz norm given by

$$
\operatorname{Lip}(T)=\sup \left\{\frac{\|T(x)-T(y)\|}{d(x, y)}: x, y \in X, x \neq y\right\} .
$$

We use the shorthand $X^{\sharp}:=\operatorname{Lip}_{0}(X, \mathbb{K})=\operatorname{Lip}_{0}(X)$, for $\mathbb{K}=\mathbb{R}$ or $\mathbb{C}$.

A molecule on $X$ is a real-valued function $m: X \longrightarrow \mathbb{R}$ with finite support that satisfies $\sum_{x \in X} m(x)=0$, the real linear space of all molecules on $X$ is denoted by $\mathcal{M}(X)$. For $x, y \in X$, the molecule $m_{x y}$ is defined by $m_{x y}=\chi_{\{x\}}-\chi_{\{y\}}$, where $\chi_{A}$ is the characteristic function of the set $A$. For $m \in \mathcal{M}(X)$, we can write $m=\sum_{j}^{n} \alpha_{j} m_{x_{j} y_{j}}$ and $\alpha_{j}$ are scalars. It's well known that $\operatorname{Lip}_{0}(X)$ has a predual, namely the space of Arens-Eells of $X$, denoted $Æ(X)[2]$, which is the completion of the space of molecules with the norm

$$
\|m\|_{Æ(X)}=\inf \left\{\sum_{j=1}^{n}\left|\alpha_{j}\right| d\left(x_{j}, y_{j}\right): m=\sum_{j=1}^{n} \alpha_{j} m_{x_{j} y_{j}}\right\} .
$$

Sawashima [17] defined the Lipschitz adjoint (or dual) between a pointed metric spaces $X$ and $Y$ with $T(0)=0$; i.e., $T \in \operatorname{Lip}_{0}(X, Y)$, as the continuous linear operator

$$
\begin{aligned}
T^{\sharp}: Y^{\sharp} & \longrightarrow X^{\sharp} \\
f & \longmapsto T^{\sharp}(f)=f \circ T,
\end{aligned}
$$

where $f \in Y^{\sharp}$. The norm of $T^{\sharp}$ is given by $\left\|T^{\sharp}\right\|=\operatorname{Lip}(T)$. If $Y=F$ is a Banach space, the restriction of $T^{\sharp}$ to $Y^{*}$ is called the Lipschitz transpose operator of $T$ and is denoted by $T^{t}$.

The notion of strongly Lipschitz $p$-nuclear operators was introduced by Chen and Zheng in [9], such that a Lipschitz operator $T: X \longrightarrow F$ is strongly Lipschitz $p$-nuclear operator if, and only if, $T$ has the form:

$$
T=\sum_{j} f_{j} \otimes y_{j}
$$


where $\left(f_{j}\right)_{j}$ in $X^{\sharp}$ and $\left(y_{j}\right)_{j}$ in $F$. The collection of all strongly Lipschitz $p$-nuclear operators from $X$ to $F$ is denoted by $\mathcal{S N}_{p}^{L}(X, F)$, and the strongly Lipschitz $p$-nuclear norm was defined as following

$$
s v_{p}^{L}(T)=\inf \left[\left(\sum_{j}\left(\operatorname{Lip}\left(f_{j}\right)\right)^{p}\right)^{\frac{1}{p}} \sup _{\left\|y^{*}\right\| \leq 1}\left(\sum_{j}\left|\left\langle y_{j}, y^{*}\right\rangle\right|^{p^{*}}\right)^{\frac{1}{p^{*}}}\right] .
$$

For $p=1$,

$$
s v_{p}^{L}(T)=\left(\sum_{j}\left(\operatorname{Lip}\left(f_{j}\right)\right)\left(\sup _{j}\left\|y_{j}\right\|\right) .\right.
$$

For $p=\infty$,

$$
s v_{p}^{L}(T)=\left(\sup _{j}\left(\operatorname{Lip}\left(f_{j}\right)\right) \sup _{\left\|y^{*}\right\| \leq 1}\left(\sum_{j}\left|\left\langle y_{j}, y^{*}\right\rangle\right|\right), \lim _{j}\left(\operatorname{Lip}\left(f_{j}\right)\right)=0 .\right.
$$

Furthermore, Chen and Zheng in [9, Theorem 2.2] prove that, $T$ is strongly Lipschitz $p$ nuclear operator if, and only if, $T$ has a factorization $T=A M_{\lambda} B$ such that the following diagram commutes:

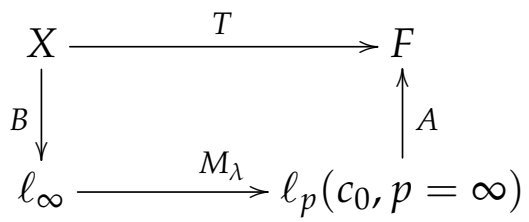

FIGURE 1. Factorization diagram of a strongly Lipschitz p-nuclear operator

where $B \in \operatorname{Lip}_{0}\left(X, \ell_{\infty}\right), M_{\lambda} \in \mathcal{L}\left(\ell_{\infty}, \ell_{p}\right)\left(\mathcal{L}\left(\ell_{\infty}, c_{0}\right), p=\infty\right)$ is the diagonal operator defined as follows (see [10, p.111]): $M_{\lambda}\left(\xi_{n}\right)=\left(\xi_{n} \lambda_{n}\right)_{n},\left(\xi_{n}\right)_{n} \in \ell_{\infty}$, with $\left\|M_{\lambda}\right\|=\|\lambda\|_{p}$, and $A \in \mathcal{L}\left(\ell_{p}, F\right)$. Moreover

$$
s v_{p}^{L}(T)=\inf \|A\|\left\|M_{\lambda}\right\| \operatorname{Lip}(B),
$$

where the infimum being extended over all factorizations as above.

The concept of strongly Lipschitz $(p, r, s)$-nuclear operators introduced and investigated by Belacel and Chen in [3] which is an extension to the notion of linear $(p, r, s)$-nuclear operators [16]. As in the linear case, the strongly Lipschitz $p$-nuclear operators coincide with the strongly Lipschitz $(p, 1, p)$-nuclear operators. The set of all strongly Lipschitz $p$-nuclear operators $\mathcal{S N}_{p}^{L}(X, F)$ is a Banach Lipschitz operator ideal [1].

In [12, Definition 2.1], Vargas and al. introduced the notion of Lipschitz compact operators, such that a Lipschitz operator $T: X \longrightarrow F, T(0)=0$ is Lipschitz compact if

$$
\left\{\frac{f(x)-f(y)}{d(x, y)}: x, y \in X, x \neq y\right\}
$$


is a relatively compact subset of $F$.

\section{Onto isometries}

In this section, we study the Lipschitz version of the isometric onto operators of $\mathcal{N}_{p}(E, F)$ [15, Sect. 3].

Theorem 3.1. Let $\left(S_{j}^{\sharp}\right)_{j}$ be a sequence of an isometric onto operators on $X^{\sharp}$, and $R$ be an isometric onto operator on F. Let $T$ be a strongly Lipschitz p-nuclear operator from $X$ into $F$ such that

$$
J(T)=J\left(\sum_{j} f_{j} \otimes y_{j}\right)=\sum_{j} \alpha_{j} S_{j}^{\sharp} f_{\varphi(j)} \otimes R y_{j},
$$

where $\left(\alpha_{j}\right)_{j}$ in $\mathbb{R},\left|\alpha_{j}\right|=1$ and $\varphi$ is a permutation on $\mathbb{N}$. Then $J$ is an isometric operator of $\mathcal{S N}_{p}^{L}(X, F)$. Proof. Assume that $T \in \mathcal{S N}_{p}^{L}(X, F)$, then $T$ has the following form

$$
T=\sum_{j} f_{j} \otimes y_{j}
$$

where $\left(f_{j}\right)_{j}$ in $X^{\sharp}$ and $\left(y_{j}\right)_{j}$ in $F$, we have

$$
\begin{aligned}
J(T) & =\sum_{j} \alpha_{j} S_{j}^{\sharp} f_{\varphi(j)} \otimes R y_{j} \\
& =\sum_{j} \tilde{f}_{j} \otimes \tilde{y}_{j} .
\end{aligned}
$$

Now,

$$
s v_{p}^{L}(T)=\inf \left(\sum_{j}\left(\operatorname{Lip}\left(f_{j}\right)\right)^{p}\right)^{\frac{1}{p}} \sup _{\left\|y^{*}\right\| \leq 1}\left(\sum_{j}\left|\left\langle y_{j}, y^{*}\right\rangle\right|^{p^{*}}\right)^{\frac{1}{p^{*}}} .
$$

Since $S_{j}^{\sharp}$ is an isometry, $\left|\alpha_{j}\right|=1$ and $\varphi$ is a permutation,

$$
\begin{aligned}
\sum_{j}\left(\operatorname{Lip}\left(\alpha_{j} S_{j}^{\sharp} f_{\varphi(j)}\right)\right)^{p} & =\sum_{j}\left|\alpha_{j}\right|^{p}\left(\operatorname{Lip}\left(S^{\sharp} f_{\varphi(j)}\right)\right)^{p} \\
& =\sum_{j}\left(\operatorname{Lip}\left(f_{\varphi(j)}\right)\right)^{p} \\
& =\sum_{j}\left(\operatorname{Lip}\left(f_{j}\right)\right)^{p}
\end{aligned}
$$

and also $R$ is an isometric onto operator on $F$, so

$$
\begin{aligned}
\sup _{\left\|y^{*}\right\| \leq 1}\left(\sum_{j}\left|\left\langle R y_{j}, y^{*}\right\rangle\right|^{p^{*}}\right)^{\frac{1}{p^{*}}} & =\sup _{\left\|y^{*}\right\| \leq 1}\left(\sum_{j}\left|\left\langle y_{j}, R^{*} y^{*}\right\rangle\right|^{p^{*}}\right)^{\frac{1}{p^{*}}} \\
& =\sup _{\left\|R^{*} y^{*}\right\| \leq 1}\left(\sum_{j}\left|\left\langle y_{j}, R^{*} y^{*}\right\rangle\right|^{p^{*}}\right)^{\frac{1}{p^{*}}} .
\end{aligned}
$$




$$
=\sup _{\left\|\tilde{y}^{*}\right\| \leq 1}\left(\sum_{j}\left|\left\langle y_{j}, \tilde{y}^{*}\right\rangle\right|^{p^{*}}\right)^{\frac{1}{p^{*}}} .
$$

Thus, $J(T)$ is strongly Lipschitz $p$-nuclear operator of $\mathcal{S N}_{p}^{L}(X, F)$ and

$$
s v_{p}^{L}(J(T))=s v_{p}^{L}(T)
$$

Theorem 3.2. Let $S$ be an isometric onto operator of $X$ into $X$ and $R$ be an isometric onto operator of $F$ into F. Assume $T$ be a strongly Lipschitz p-nuclear operator from $X$ into $F$ such that

$$
J(T)=R T S \text {. }
$$

Then $J(T)$ is an isometric onto operator of $\mathcal{S N}_{p}^{L}(X, F)$.

Proof. Let $T \in \mathcal{S N}_{p}^{L}(X, F)$, then $T$ has the following representation

$$
T=\sum_{j} f_{j} \otimes y_{j}
$$

so

$$
\begin{aligned}
J(T) x & =R T S x \\
& =R\left(\sum_{j}\left(f_{j}(S x)\right) y_{j}\right. \\
& =\sum_{j}\left(S^{\sharp}\left(f_{j} x\right)\right) R y_{j}
\end{aligned}
$$

Thus,

$$
J(T)=\sum_{j} S^{\sharp} f_{j} \otimes R y_{j} .
$$

Since $S$ is an isometric onto operator of $X$, then $S^{\sharp}$ is an isometric onto operator of $X^{\sharp}$, and $R$ is an isometric onto operator of $F$. Now, we show that $J$ preserves norm,

$$
\begin{aligned}
s v_{p}^{L}(J(T)) & =\inf \left(\sum_{j}\left(\operatorname{Lip}\left(S^{\sharp} f_{j}\right)\right)^{p}\right)^{\frac{1}{p}} \sup _{\left\|y^{*}\right\| \leq 1}\left(\sum_{j}\left|\left\langle y_{j}, R^{*} y^{*}\right\rangle\right|^{p^{*}}\right)^{\frac{1}{p^{*}}} \\
& =\inf \left(\sum_{j}\left(\operatorname{Lip}\left(f_{j}\right)\right)^{p}\right)^{\frac{1}{p}} \sup _{\left\|R^{*} y^{*}\right\| \leq 1}\left(\sum_{j}\left|\left\langle y_{j}, R^{*} y^{*}\right\rangle\right|^{p^{*}}\right)^{\frac{1}{p^{*}}} \\
& =\inf \left(\sum_{j}\left(\operatorname{Lip}\left(f_{j}\right)\right)^{p}\right)^{\frac{1}{p}} \sup _{\left\|\tilde{y}^{*}\right\| \leq 1}\left(\sum_{j}\left|\left\langle y_{j}, \tilde{y}^{*}\right\rangle\right|^{p^{*}}\right)^{\frac{1}{p^{*}}} \\
& =s v_{p}^{L}(T) .
\end{aligned}
$$

Thus,

$$
s v_{p}^{L}(J(T))=s v_{p}^{L}(T) .
$$


To show that $J$ is onto, let $T=\sum_{j} f_{j} \otimes y_{j}$ be a strongly Lipschitz $p$-nuclear operator, and $\tilde{T}=\sum_{j} f_{j} S^{-1} \otimes R^{-1} y_{j}$, so

$$
J(\tilde{T})=\sum_{j} f_{j} \otimes y_{j}=T,
$$

this implies that $J$ is onto.

Hence, $J$ is an isometric onto operator of $\mathcal{S N}_{p}^{L}(X, F)$.

\section{Lipschitz operators, where $F=\ell_{p^{*}}$}

In this section, we characterize the Lipschitz operators that takes values in $\ell_{p^{*}}$ whose linear analogue has found in $[15$, Sect. 4].

Theorem 4.1. Let $2 \leq p<\infty$ and $T$ be a Lipschitz operator from $X$ into $\ell_{p^{*}}$. Then $T$ is a strongly Lipschitz p-nuclear operator from $X$ into $\ell_{p^{*}}$, with

$$
s v_{p}^{L}(T)=\left(\sum_{j}\left|\lambda_{j}\right|^{p}\right)^{\frac{1}{p}}
$$

where $\left(\lambda_{j}\right)_{j} \in \ell_{p^{*}}$.

Proof. Given the fact that each Lipschitz operator can be factored as [18, Theorem 2.2.4]

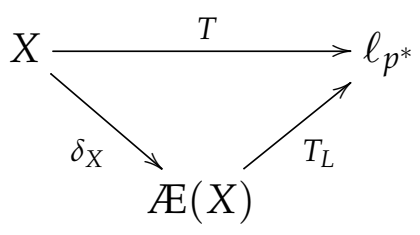

FIGURE 2. Factorization diagram of Lipschitz operator

where $\delta_{X}(x)=m_{x 0}$ is an isometric embending from $X$ into $Æ(X)$ and $T_{L}$ is unique linear operator from $Æ(X)$ to $F$ (for more details see [18, p. 38-43]). Since $T_{L}$ is $p$-nuclear operator [15, Sect.4] and $T=T_{L} \circ \delta_{X}$, then by [1, Proposition 3.2] $T$ is a strongly Lipschitz $p$-nuclear operator with $s v_{p}^{L}(T)=v_{p}\left(T_{L}\right)$, by means of [15, Theorem 6] we get

$$
s v_{p}^{L}(T)=\left(\sum_{j}\left|\lambda_{j}\right|^{p}\right)^{\frac{1}{p}} .
$$

Using Theorem 4.1, we obtain the following results:

Corollary 4.1. Let $2 \leq p<\infty$ and $T$ be a strongly Lipschitz $p$-nuclear operator from $X$ into $\ell_{p^{*}}$. Then $T$ has representation for which the infimum is attained. 
We will give in the next corollary the same result as [9, Theorem 2.1] with $E$ only a Banach space.

Corollary 4.2. Let $2 \leq p<\infty$ and $T$ be a linear operator from $E$ into $\ell_{p^{*}}$. Then

$$
s v_{p}^{L}(T)=v_{p}(T) .
$$

Proof. From [15, Theorem 6] and by Theorem 4.1 we get

$$
s v_{p}^{L}(T)=v_{p}(T) .
$$

Corollary 4.3. The space $\mathcal{S} \mathcal{N}_{p}^{L}\left(X, \ell_{p^{*}}\right)$ and $\ell_{p^{*}}^{L, w^{*}}\left(X^{\sharp}\right)$ are isometrically isomorphic.

Proof. There is an isomorphism isometry between $\operatorname{Lip}_{0}\left(X, \ell_{p^{*}}\right)$ and $\ell_{p^{*}}^{L, w^{*}}\left(X^{\sharp}\right)$ [3, Lemma 2.4], and $\operatorname{Lip}_{0}\left(X, \ell_{p^{*}}\right)=\mathcal{S N}_{p}^{L}\left(X, \ell_{p^{*}}\right)$ Theorem 4.1. Hence $\mathcal{S N}_{p}^{L}\left(X, \ell_{p^{*}}\right)$ and $\ell_{p^{*}}^{L, w^{*}}\left(X^{\sharp}\right)$ are isometrically isomorphic.

Corollary 4.4. Let J be an isometric onto operator of $\mathcal{S N}_{p}^{L}\left(X, \ell_{p^{*}}\right)$. Then J is Lipschitz compact operator.

Proof. It's known that $\operatorname{Lip}_{0 K}\left(X, \ell_{p^{*}}\right) \subset \operatorname{Lip}_{0}\left(X, \ell_{p^{*}}\right)$ [12] and Then by Theorem 4.1

$\operatorname{Lip}_{0 K}\left(X, \ell_{p^{*}}\right)=\mathcal{S N}_{p}^{L}\left(X, \ell_{p^{*}}\right)$.

The next theorem we show that an isometric onto operators of a Lipschitz operator ideal preserves the rank.

Theorem 4.2. Let $2 \leq p<\infty$ and $J$ be an isometric onto operator of $\mathcal{S N}_{p}^{L}\left(X, \ell_{p^{*}}\right)$. Then J preserves the rank.

Proof. It's known that an isometry on $\ell_{p}$ preserve the support. We will use this in our proof. If $T \in \mathcal{S N}_{p}^{L}\left(X, \ell_{p^{*}}\right)$, then by Theorem 4.1

$$
T=\sum_{j} \lambda_{j} f_{j} \otimes \delta_{j}
$$

Therefore $J(T) \in \mathcal{S N}_{p}^{L}\left(X, \ell_{p^{*}}\right)$,

$$
J(T)=\sum_{j} \xi_{j} g_{j} \otimes \delta_{j}
$$

where $\left\|\left(\lambda_{j}\right)\right\|_{p}=\left\|\left(\xi_{j}\right)\right\|_{p}$ and $\operatorname{Lip}\left(f_{j}\right)=\operatorname{Lip}\left(g_{j}\right)$. This produces

$$
\hat{J}: \ell_{p} \longrightarrow \ell_{p}
$$

where $\hat{J}\left(\lambda_{j}\right)=\left(\xi_{j}\right)_{j}$. Clearly $\hat{J}$ is an isometric onto operator on $\ell_{p}$. So it preserve the support. That is $\operatorname{supp}\left(\lambda_{j}\right) \cap \operatorname{supp}\left(\xi_{j}\right)=\varnothing$, then $\operatorname{supp}\left(\hat{J}\left(\lambda_{j}\right)\right) \cap \operatorname{supp}\left(\hat{J}\left(\xi_{j}\right)\right)=\varnothing$. Indeed

$$
\begin{aligned}
\left\|\hat{J}\left(\lambda_{j}\right)+\hat{J}\left(\xi_{j}\right)\right\|_{p}^{p} & =\left\|\hat{J}\left(\lambda_{j}+\xi_{j}\right)\right\|_{p}^{p} \\
& =\left\|\lambda_{j}+\xi_{j}\right\|_{p}^{p}
\end{aligned}
$$




$$
\begin{aligned}
& =\left\|\left(\lambda_{j}\right)\right\|_{p}^{p}+\left\|\left(\xi_{j}\right)\right\|_{p}^{p} \\
& =\|\left(\hat { J } ( \lambda _ { j } ) \| _ { p } ^ { p } + \| \left(\hat{J}\left(\xi_{j}\right) \|_{p}^{p} .\right.\right.
\end{aligned}
$$

Since $\operatorname{rank}(J(T))=\left|\operatorname{supp}\left(\hat{J}\left(\xi_{j}\right)\right)\right|=\left|\operatorname{supp}\left(\lambda_{j}\right)\right|=\operatorname{rank}(T)$. So it's preserves rank.

For $f \in X^{\sharp}$ and $y \in F$ (Banach space), we define the one rank operator $f \otimes y: X \longrightarrow F$ by $(f \otimes y)(x)=f(x) y$. The rank one operator is called an atom.

The next corollary is an immediate consequence of Theorem 4.2.

Corollary 4.5. Let $J$ be an isometric onto operator of $\mathcal{S N}_{p}^{L}\left(X, \ell_{p^{*}}\right)$. Then J preserves atoms.

Now, we give in the next theorem the main result of this section. We adapt the proof in [13, Theorem 3.3] to Lipschitz situation.

Theorem 4.3. Let $J: \mathcal{S N}_{p}^{L}\left(X, \ell_{p^{*}}\right) \longrightarrow \mathcal{S N}_{p}^{L}\left(X, \ell_{p^{*}}\right)$. If J preserves rank and that preserves the basic atoms, then the following are equivalent:

(1) $J$ is an isometric onto operator.

(2) There exist two isometric onto operators:

$S: X^{\sharp} \rightarrow X^{\sharp}$ and $W: \ell_{p^{*}} \rightarrow \ell_{p^{*}}$ and a sequence $\left(a_{j}\right)_{j},\left|a_{j}\right|=1$ for all $j$, with

$$
J(T)=\sum_{j} a_{j} S f_{j} \otimes W \delta_{j} .
$$

Proof. $1 \Rightarrow 2$. Let $J$ be an isometric onto operator.

Step 1: Let $M=\left\{f \otimes \delta_{1}: f \in X^{\sharp}\right\}$. Then

$$
J(M)=\left\{g \otimes \delta_{j}: \text { for fixed } j, \forall g \in X^{\sharp}\right\} .
$$

Assume $J\left(f_{1} \otimes \delta_{1}\right)=\hat{f}_{1} \otimes \delta_{j_{1}}$ and $J\left(f_{2} \otimes \delta_{1}\right)=\hat{f}_{2} \otimes \delta_{j_{2}}$ where $\hat{f}_{1}, \hat{f}_{2} \in X^{\sharp}$, and $\delta_{j_{1}} \neq \delta_{j_{2}}$. Then for some $g \in X^{\sharp}$ and $j \in \mathbb{N}$ :

$$
\begin{aligned}
\hat{f}_{1} \otimes \delta_{j_{1}}+\hat{f}_{2} \otimes \delta_{j_{2}} & =J\left(f_{1} \otimes \delta_{1}\right)+J\left(f_{1} \otimes \delta_{2}\right) \\
& =J\left(f_{1} \otimes \delta_{1}+f_{1} \otimes \delta_{2}\right) \\
& =J\left(f_{1} \otimes\left(\delta_{1}+\delta_{2}\right)\right) \\
& =g \otimes \delta_{j}
\end{aligned}
$$

This implies $\hat{f}_{1} \otimes \delta_{j_{1}}+\hat{f}_{2} \otimes \delta_{j_{2}}=g \otimes \delta_{j}$ with $\delta_{j_{1}} \neq \delta_{j_{2}}$. But $f_{1} \otimes \delta_{1}+f_{2} \otimes \delta_{1}=\left(f_{1}+f_{2}\right) \otimes \delta_{1}$ is a basic atom, and $J$ preserves basic atoms which is a contradiction because $\hat{f}_{1} \otimes \delta_{j_{1}}+\hat{f}_{2} \otimes \delta_{j_{2}}$ is not a basic atom, since $\delta_{j_{1}} \neq \delta_{j_{2}}$. So $J\left(f \otimes \delta_{1}\right)=g \otimes \delta_{j}$ for fixed $j \in \mathbb{N}$. Similarly for $\delta_{2}, \ldots$

Step 2: Let $J\left(f \otimes \delta_{1}\right)=g \otimes \delta_{j}$. Define $W: \ell_{p^{*}} \rightarrow \ell_{p^{*}}, W \delta_{1}=\delta_{j}, W$ it permutes the basis $\left(\delta_{j}\right)$, so $W$ is an onto and $W \delta_{1}=\delta_{\varphi(j)}$, where $\varphi$ is a permutation on the set of natural numbers $\mathbb{N}$. Since $J$ is an isometric, then $W$ is an isometric. Similarly for $\delta_{2}, \ldots$ 
Step 3: Assume $f \otimes \delta_{1}+f \otimes \delta_{2}=f \otimes\left(\delta_{1}+\delta_{2}\right)$ be a basic atom (1-rank operator). Let $J\left(f \otimes \delta_{1}\right)=g \otimes \delta_{\varphi(1)}$ and $J\left(f \otimes \delta_{2}\right)=h \otimes \delta_{\varphi(2)}$, such that $g \neq h$. Then $J\left(f \otimes\left(\delta_{1}+\delta_{2}\right)\right)=$ $g \otimes \delta_{\varphi(1)}+h \otimes \delta_{\varphi(2)}$, Since $J$ is an isometry, then $\operatorname{Lip}(f)=\operatorname{Lip}(g)=\operatorname{Lip}(h)$, but this implies that either $g, h$ are independent or dependent i.e $g= \pm h$. If $g, h$ are independent, then $J(f \otimes$ $\left.\left(\delta_{1}+\delta_{2}\right)\right)=g \otimes \delta_{\varphi(1)}+h \otimes \delta_{\varphi(2)}$ is two rank operator which is a contradiction, because $J$ preserves rank. Hence $J\left(f \otimes \delta_{1}\right)=a_{1} g \otimes \delta_{\varphi(1)}$ and $J\left(f \otimes \delta_{2}\right)=a_{2} g \otimes \delta_{\varphi(2)}$, with $\left|a_{j}\right|=1$. Similarly we prove

$$
\left\{J\left(f \otimes \delta_{j}\right): j \in \mathbb{N}\right\}=\left\{a_{j} g \otimes \delta_{\varphi(j)}:\left|a_{j}\right|=1, j \in \mathbb{N}\right\} .
$$

Step 4: Define $S: X^{\sharp} \rightarrow X^{\sharp}, S(f)=g$, where $J\left(f \otimes \delta_{j}\right)=g \otimes a_{j} \delta_{\varphi(j)}$ with $\left|a_{j}\right|=1$. It's easy to check that $S$ is well-defined isometric linear operator. Let $g \in X^{\sharp}: J\left(f \otimes \delta_{\varphi^{-1}(j)}\right)=a_{j} g \otimes \delta_{j}$, where $\varphi: \mathbb{N} \longrightarrow \mathbb{N}$ is one-to-one and onto map on the set of natural numbers $\mathbb{N}$. Since $J$ is onto, then for some $g=S(f)$. This implies $S$ is onto.

We show now that $J(T)=\sum_{j} a_{j} S f_{j} \otimes W \delta_{j}$. We have

$$
J\left(\sum_{j} f_{j} \otimes \delta_{j}\right)=\sum_{j} J\left(f_{j} \otimes \delta_{j}\right)=\sum_{j} a_{j} g_{j} \otimes \delta_{\varphi(j)}=\sum_{j} a_{j} S f_{j} \otimes W \delta_{j} .
$$

$2 \Rightarrow 1$. Let $T \in \mathcal{S N}_{p}^{L}\left(X, \ell_{p^{*}}\right)$. Since $S, W$ is an isometry we have

$$
J(T)=\sum_{j} a_{j} S f_{j} \otimes W \delta_{j}=\sum_{j} a_{j} g_{j} \otimes \delta_{\varphi(j)}=\tilde{T},
$$

where $\operatorname{Lip}\left(f_{j}\right)=\operatorname{Lip}\left(g_{j}\right)$, with

$$
s v_{p}^{L}(J(T))=s v_{p}^{L}(\tilde{T})=\left(\sum_{j}\left|\lambda_{j}\right|^{p}\right)^{\frac{1}{p}}=s v_{p}^{L}(T) .
$$

Thus $J$ is an isometry.

Now we show that $J$ is onto, let $T=\sum_{j} f_{j} \otimes \delta_{j}$ be a strongly Lipschitz $p$-nuclear operator, where $f_{j}=a_{j} g_{j}$, with $\left|a_{j}\right|=1$, let $\tilde{T}=\sum_{j} S^{-1} g_{j} \otimes \delta_{\varphi^{-1}(j)}$, so

$$
J(\tilde{T})=\sum_{j} a_{j} g_{j} \otimes \delta_{j}=\sum_{j} f_{j} \otimes \delta_{j}=T,
$$

this implies that $J$ is onto.

\section{Acknowledgments.}

The authors wish to thank Professor Roshdi Khalil, Department of Mathematics, Amman, 11942 , Jordan, for his support for this paper and to anonymous referee for many important suggestions in order to make paper more coherent. They acknowledge the support of the General Direction of Scientific Research and Technological Development (DGRSDT), Algeria. 


\section{References}

[1] D. Achour, P. Rueda, E.A. Snchez-Prez, and R. Yahi, Lipschitz operator ideals and the approximation property, J. Math. Anal. Appl., 436 (2016), 217-236.

[2] R.F. Arens, J. Jr. Eells, On embedding uniform and topological spaces, Pac. J. Math., 6 (1956), 397-403.

[3] A. Belacel, D. Chen, Lipschitz ( $\mathrm{p}, \mathrm{r}, \mathrm{s})$-integral operators and Lipschitz $(\mathrm{p}, \mathrm{r}, \mathrm{s})$-nuclear operators, J. Math. Anal. Appl. 461 (2018), 1115-1137.

[4] A. Belacel and Kh. Bey, Strongly Lipschitz up-nuclear operators, Moroccan J. of Pure and Appl. Anal. 5(1) (2019), 22-30.

[5] M.G. Cabrera-Padilla, A. Jimnez-Vargas, and M. Villegas-Valle Cillos, A survey on isometries between Lipschitz spaces. The Moroccan Andalusian Meeting on algebras and thier applications, (2018), 51-88.

[6] J.A. Chávez-Domínguez, Duality for Lipschitz p-summing operators, J. Funct Anal., 261 (2011), 387-407.

[7] J.A. Chávez-Domínguez, Lipschitz (q, p)-mixing operators, Proc. Am. Math. Soc., 140 (2012), 3101-3115.

[8] D. Chen, B. Zheng, Remarks on Lipschitz p-summing operators, Proc. Am. Math. Soc., 139 (8) (2011), 28912898.

[9] D. Chen, B. Zheng, Lipschitz p-integral operators and Lipschitz p-nuclear operators, Nonlinear Anal., 75 (2012), 5270-5282.

[10] J. Diestel, H. Jarchow, and A. Tonge, Absolutely Summing Operators, Cambridge University Press, Cambridge, (1995).

[11] J.D. Farmer, W.B, Johnson, Lipschitz p-summing operators. Proc. Am. Math. Soc., 137(9) (2009), 2989-2995.

[12] A. Jimnez-Vargas, J.M. Sepulcre, and M. Villegas-Vallecillos, Lipschitz compact operators, J. Math. Anal. Appl., 415(2) (2014), 889-901.

[13] R. Khalil, I. Adarwi, Isometries of p-nuclear type operators, J. Math. Comput. Sci, 5 (2015), 91-98.

[14] R. Khalil, A. Salah, Isometries of certains operator spaces Proc. An. Math.Soc, 2003, 14731481.

[15] R. Khalil, A. Yousef, Isometries of p-nuclear operators, J. Comput. Anal. Appl., 16(2) (2014), 368-374.

[16] A. Pietsch, Operator ideals, North-Holland publishing company, Amsterdam, 1980.

[17] I. Sawashima, Methods of Lipschitz Duals, Lecture Notes Ec. Math Sust, vol. 419 (1975), 247-259.

[18] N. Weaver, Lipschitz Algebras, World Scientific Publishing Co., Singapore, (1999). 